\title{
Increased risk of non-hematological cancer in young patients with aortic stenosis: a retrospective cohort study
}

\author{
Walid Saliba 1,2, Tamir Bental ${ }^{3}$, Yaron Shapira ${ }^{3}$, Shmuel Schwartzenberg ${ }^{3}$, Alex Sagie ${ }^{3}$, Moti Vaturi ${ }^{3}$, \\ Salim Adawi ${ }^{2,4}$, Alexander Fuks ${ }^{4}$, Ami Aronheim ${ }^{5}$ and Avinoam Shiran ${ }^{2,4^{*}(\text { C) }}$
}

\begin{abstract}
Background: We have previously reported an increased risk for non-hematological malignancies in young patients with moderate or severe aortic stenosis (AS). These findings were the result of a post-hoc analysis from a large echocardiography database and needed verification. Our aim was to determine, using a different study population, whether young patients with AS are at increased risk for cancer.
\end{abstract}

Methods: A large echocardiographic database was used to identify patients (age $\geq 20$ years) with moderate or severe AS (study group) and patients without aortic stenosis (comparative group). The new occurrence of non-hematological malignancies was determined after the index date (first echo with moderate or severe AS or first recorded echo in the control group).

Results: The final study group included 7013 patients with AS and 98,884 without AS. During a median follow-up of 6.9 years (3.0-11.1) there were 10,705 new cases of non-hematological cancer. The crude incidence rate of cancer was higher in AS compared to non-AS patients (22.3 vs. 13.7 per 1000 patient-year, crude HR 1.58 (95\%Cl 1.46-1.71). After adjustment for relevant covariates, there was no difference between groups (HR 0.93, 95\% Cl 0.86-1.01). Only patients in the lowest age quartile (20-49.7 years), had an increased adjusted risk of cancer (HR 1.91,95\% Cl 1.08-3.39). The HR for the risk of cancer associated with AS was inversely proportional to age $(P<0.001$ for the interaction between AS and age).

Conclusions: Young patients with moderate or severe AS may have an increased risk for cancer. Cancer surveillance should be considered for young patients with AS.

Keywords: Aortic stenosis, Cancer, Age, Risk

\section{Introduction}

Reverse cardio-oncology has been recently proposed as a new field, dealing with inherent cancer risk in patients with cardiovascular disease [1]. Cancer and cardiovascular disease share similar risk factors, including smoking, obesity, diabetes mellitus, hyperlipidemia and a sedentary

\footnotetext{
${ }^{*}$ Correspondence: av.shiran@gmail.com

${ }^{4}$ Department of Cardiology, Lady Davis Carmel Medical Center, 7 Michal Street, 3436212 Haifa, Israel
}

Full list of author information is available at the end of the article lifestyle, which may partly explain this phenomenon [2, $3]$. Increased risk of cancer has been reported in patients with heart failure (HF) [4-6]. Compared to patients without HF, community patients with HF were found to have a $68 \%$ higher adjusted risk of developing cancer which was associated with higher mortality, during an average follow-up of 7.7 years [4]. Similarly, patients who developed HF after acute myocardial infarction had an increased adjusted risk of cancer [5]. In a Danish population study, increased risk of cancer was reported in all 
age groups of outpatients with chronic HF, but a surveillance bias could not be ruled out [6]. To determine whether causal relationship exists between HF and the development of cancer, Meijers et al. used a mouse model of myocardial infarction and HF, and showed increased intestinal tumor growth, proportional to the amount of scar tissue in the left ventricle and inversely proportional to left ventricular ejection fraction [7]. This effect was mediated by cardiac secreted proteins. In another study, myocardial infarction was shown to accelerate breast cancer progression and mortality in mice and humans through an epigenetic switch in monocytes to an immunosuppressive phenotype [8].

We have previously shown in a transverse aortic constriction (TAC) murine model, that early cardiac remodeling secondary to pressure overload, without HF, is associated with increased risk of cancer [9]. In this model, cardiac remodeling was associated with increased tumor growth and metastasis seeding, which was suggested to be mediated by periostin, a matrix protein that enhances cancer cell proliferation. In that study, using a large retrospective echocardiographic database and the national cancer registry we have shown that moderate or severe aortic stenosis (AS) is associated with a significantly increased risk of non-hematologic malignancies in patients younger than 60 years old, but not in older patients [9]. Our findings suggested that age may modify the effect of AS on non-hematologic malignancies. These findings were the result of post-hoc analysis and needed confirmation by an independent data set. Our aim in the present study, was to examine the effect of AS on the risk of non-hematologic malignancies in different age groups, using data from a different patient population.

\section{Methods}

\section{Source of data}

In the present study we used the echocardiography database of a large tertiary hospital, the Rabin Medical Center from the Clalit Health Services (CHS), which includes both inpatients and outpatients. CHS hospitals provide health services for all patients, including patients from other health maintenance organizations (HMO). This analysis was restricted to CHS members, for whom we have full access to clinical data including outcomes data and other relevant confounders. CHS provides inclusive health care for more than half of the Israeli population. Health care coverage in Israel is mandatory according to the National Health Insurance Law (1995) and is provided by four groups akin to non-profit health HMO. All members of the different HMOs have a similar health insurance plan and similar access to health services. The electronic medical record database of CHS includes data from multiple sources: records of primary care physicians, community specialty clinics, hospitalizations, laboratories, and pharmacies. A registry of chronic disease diagnoses is compiled from these data sources. Diagnoses are captured in the registry by diagnosis-specific algorithms, employing International Classification of Diseases Ninth revision (ICD-9) code reading, laboratory test results and disease-specific drug usage. A record is kept of the data-sources and dates used to establish the diagnosis, with the earliest recorded date, from any source, considered to be the defining date of diagnosis. This process of capturing data into the registry reassures that a specific type of cancer diagnosed after the index date is not a recurrence or continuation of an original cancer diagnosed before the index date. A number of high-quality, population-based studies have been conducted based on the data retrieved from $\mathrm{CHS}$ database [10-12].

The electronic medical records database of the CHS is periodically updated with data from the Israel National Cancer Registry (INCR). Cancer reporting by hospitals, pathology and cytology laboratories and other health care providers has been mandatory since 1982 . The INCR collects data on all malignant, in-situ and invasive tumors (excluding basal and squamous cell carcinomas of the skin). The completeness of the INCR is estimated at $96.8 \%$ for invasive solid malignancies [13]. The current retrospective cohort study comprised only invasive nonhematological malignancies (invasive solid tumors).

\section{Selection of study population}

Eligible patients were CHS adult members age $\geq 20$ years who underwent echocardiography at Rabin Medical Center, between January 1st, 2005 and December 31st, 2019 , and were found to have either no documentation of any degree of AS or at least one test showing moderate or severe AS. Patients were excluded if they were not members of CHS, and if they had coronary artery bypass surgery, or they had aortic or mitral valve replacement before the index date. The first dated echo showing at least moderate AS was used to define the index date of the exposure group (AS group), and the first dated echo of patients without evidence of any degree of AS was used to define the index date of the comparative group (no AS). The two groups were followed from the index date through October 30, 2020, for the occurrence of non-hematological cancer.

The study was approved by the Institutional Review Board (IRB) and conducted in accordance with the Declaration of Helsinki.

\section{Echocardiography and study variables}

A complete transthoracic echocardiographic study was performed using standard views and techniques 
according to established guidelines [14]. The diagnosis of AS was based on 2-dimmensional echocardiographic aortic valve anatomy, trans-aortic Doppler gradients and aortic valve area (AVA), with moderate or severe AS defined as AVA $\leq 1.5 \mathrm{~cm}^{2}$, and no AS defined as AVA $>2 \mathrm{~cm}^{2}$ or maximal aortic velocity $<2.5 \mathrm{~m} / \mathrm{sec}$ [15]. AVA was calculated using the continuity equation.

In addition, for each patient the following baseline data were retrieved from the computerized database of the CHS: demographic and other descriptive variables, smoking status (ever, never), alcohol abuse, obesity, socioeconomic status (SES, based on the SES score of the clinic neighborhood as defined by the Israeli Central Bureau of Statistics), presence of selected chronic medical conditions, cancer risk factors and medication use of selected drug categories. SES had missing values; hence, this variable was used in the analyses as categorical variables that include a category of missing values.

The study outcome was defined as the occurrence of invasive non-hematologic malignancies at all sites, based on combined data from multiple sources in the CHS databases and data from the Israeli National Cancer Registry (INCR).

\section{Statistical methods}

Statistical analyses were performed using IBM SPSS Statistics 24.0 (IBM, New York, NY), and SAS version 9.3 software. For all analyses, $P<0.05$ for the 2-tailed tests was considered statistically significant. Continuous variables are summarized with mean $\pm \mathrm{SD}$, and categorical variables are presented as numbers and proportions. Comparisons of baseline characteristics, between patients with AS and patients without AS, were performed using the chi-square test for categorical variables and using student $\mathrm{t}$-test for continuous variable.

The annualized incidence rate of invasive solid tumor was estimated by dividing the number of incident cases by the total follow up time and was expressed as number per 1000 person-year of observation. Because time to invasive non-hematologic malignancies faces the competing risk of mortality, we used cumulative incidence function (CIF) to estimate the distribution of time to reach invasive non-hematologic malignancies [16]. The Gray's test for equality of CIF was used to compare curves of patients with AS and without AS. Cox proportional hazard regression models were used to estimate the crude and the adjusted hazard ratio (HR) for the association between AS and non-hematologic malignancies. The main Cox multivariable model was adjusted for age (continuous variable), sex, ethnicity, socioeconomic status, smoking, alcohol abuse, obesity, diabetes mellitus, previous history of cancer and aspirin and statin use. An interaction between AS status and age was tested by including an interaction factor of both variables into the multivariable Cox regression model. In addition, we performed the following analyses: i) To assess the robustness of our results we repeated the multivariable analysis and performed further adjustment for additional variables that were not accounted for in the main analysis, including; heart failure, ischemic heart disease, peripheral vascular disease, previous stroke, atrial fibrillation, and year of study entry. ii) To avoid possible biases, we restricted the analysis to patients without a history of cancer, since they can retain their propensity for other cancer, iii) A one-year landmark analysis was performed in order to assess the effect of possible surveillance bias shortly after AS diagnosis. This analysis excluded patients with cancer diagnosed during the first year, and was restricted to patients with greater than 1 year of follow-up.

\section{Results \\ Study population}

The final study population included 105,897 patients. AS (moderate or severe) was present in 7013 patients, and 98,884 did not have AS (Fig. 1). The study group demographic and clinical characteristics are summarized in Table 1. Patients with AS were older and more likely to have previous cancer, comorbidities, HF and cardiovascular disease. Severe AS was present in 4125/7013 patients with AS (58.8\%). Moderate or severe AS was present in 183/26,476 (0.7\%) patients in the lowest age quartile (20-49.7 years) and $4662 / 26,469(18 \%)$ of patients in the highest age quartile ( $>74.7$ years).

\section{Risk of cancer and age}

Overall, there were 10,705 new cases of non-hematological cancer during a median follow-up of 6.9 years (IQR 3.0-11.1) (Fig. 1, Table 2). The cumulative incidence of cancer and mortality among AS and non-AS patients is shown in Fig. 2. The curves diverge shortly after the index echo, showing a higher incidence of cancer and mortality in AS. However, afterward the CIF curve for non-hematological malignancy crosses between the two cohorts (Fig. 2a). Indeed, a test of the sub-distribution hazards proportionality assumption showed that this assumption was violated. The crude incidence rate of cancer was higher in AS as compared to non-AS patients (22.7 vs. 13.7 per 1000 patient-years, crude HR 1.58 (95\% CI 1.46-1.71). But after adjustment for age, sex, ethnicity, socioeconomic status, smoking, alcohol abuse, obesity, diabetes mellitus, previous history of cancer and aspirin and statin use, there was no difference between the two 


\section{Eligible patients:}

130,911 adults (age $\geq 20$ years) who underwent echocardiography between 01.01.2005 and 31.12.2019:

119,696 (91.4\%) with no documentation of any degree of AS 2,771 (2.1\%) with mild AS $8,444(6.5 \%)$ with at least one test showing moderate or severe AS

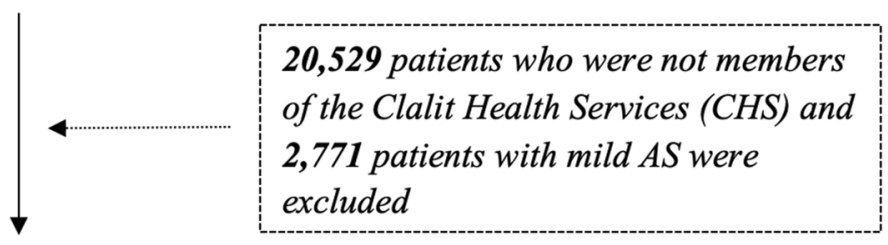

107,611 CHS members:

100,319 (93.2\%) had No AS

$7,292(6.8 \%)$ had moderate or severe AS

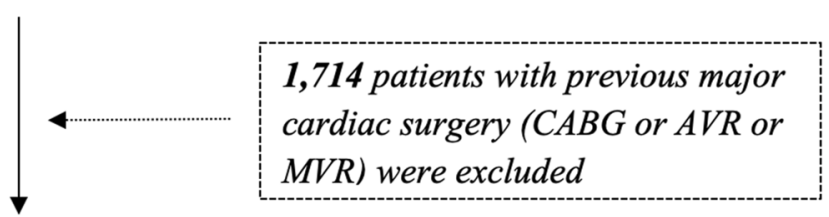

$\mathbf{1 0 5 , 8 9 7}$ CHS members with age $\geq 20$ years were included in the final analysis, of them:

$\mathbf{9 8 , 8 8 4}(93.4 \%)$ had No AS

$7,013(6.6 \%)$ had moderate or severe AS

\begin{tabular}{|l|}
\hline \multicolumn{1}{|c|}{} \\
Follow-up through 30.10.2020 \\
$\mathbf{1 0 , 7 0 5}$ new non-hematological cancers \\
occurred during a median follow-up of \\
6.9 (IQR, 3.0-11.1) years. IR = 14.0 per \\
1000 person-years
\end{tabular}

Fig. 1 Study population selection flowchart. $A S=$ aortic stenosis, $A V R=$ aortic valve replacement, $C A B G=$ coronary artery bypass grafting, $\mathrm{IQR}=$ interquartile range, $\mathrm{IR}=$ incidence rate, $\mathrm{MVR}=$ mitral valve replacement

groups (HR 0.93, 95\% CI 0.86-1.01). The HR for the risk of cancer associated with AS was inversely proportional to age $(P<0.001$ for the interaction between AS and age) (Fig. 3). Stratification analysis by age quartiles, revealed a significantly higher risk of cancer in the lowest age quartile (age 20-49.7 years), adjusted HR 1.91, 95\% CI
1.08-3.39 (Table 2). Similarly, an increased cumulative incidence of cancer in AS was observed only in the lowest age quartile (Fig. 4). In this group of patients, the proportional hazard assumption was not violated.

Since bicuspid aortic valve (BAV) is more frequent in younger patients with AS, we examined the association 
Table 1 Baseline demographic and clinical characteristics of the entire study population according to AS status

\begin{tabular}{|c|c|c|c|c|}
\hline & $\begin{array}{l}\text { All } \\
(n=105,897)\end{array}$ & $\begin{array}{l}\text { No AS } \\
(n=98,884)\end{array}$ & $\begin{array}{l}\text { Moderate or severe AS } \\
(n=7013)\end{array}$ & $P$ value \\
\hline Age & $61.1 \pm 17.5$ & $60.0 \pm 17.3$ & $77.1 \pm 11.4$ & $<0.001$ \\
\hline Sex & & & & 0.883 \\
\hline Males & $52,655(49.7 \%)$ & $49,162(49.7 \%)$ & $3493(49.8 \%)$ & \\
\hline Females & $53,242(50.3 \%)$ & $49,722(50.3 \%)$ & $3520(50.2 \%)$ & \\
\hline Ethnicity & & & & $<0.001$ \\
\hline Arabs & $7314(6.9 \%)$ & $7008(7.1 \%)$ & $306(4.4 \%)$ & \\
\hline Jews & $98,583(93.1 \%)$ & $91,876(92.9 \%)$ & $6707(95.6 \%)$ & \\
\hline $\mathrm{SES}^{\mathrm{a}}$ & & & & 0.458 \\
\hline Low & $17,464(16.5 \%)$ & $16,291(16.5 \%)$ & $1173(16.7 \%)$ & \\
\hline Middle & $53,310(50.3 \%)$ & $49,820(50.4 \%)$ & $3481(49.6 \%)$ & \\
\hline High & $33,175(31.3 \%)$ & $30,936(31.3 \%)$ & $2239(32.0 \%)$ & \\
\hline \multicolumn{5}{|l|}{ Comorbidities and risk factors } \\
\hline Smoking & $38,328(36.2 \%)$ & $35,833(36.2 \%)$ & $2495(35.6 \%)$ & 0.266 \\
\hline Alcohol abuse & $1052(1.0 \%)$ & $992(1.0 \%)$ & $60(0.9 \%)$ & 0.238 \\
\hline Obesity & $25,451(24.0 \%)$ & $23,171(23.4 \%)$ & $2280(32.5 \%)$ & $<0.001$ \\
\hline Previous cancer & $18,725(17.7 \%)$ & $16,957(17.1 \%)$ & $1768(25.2 \%)$ & $<0.001$ \\
\hline Diabetes & $26,830(25.3 \%)$ & $23,976(24.2 \%)$ & $2854(40.7 \%)$ & $<0.001$ \\
\hline Hypertension & $54,362(51.3 \%)$ & $48,681(49.2 \%)$ & $5681(81.0 \%)$ & $<0.001$ \\
\hline Congestive heart failure & $11,211(10.6 \%)$ & $9095(9.2 \%)$ & $2116(30.2 \%)$ & $<0.001$ \\
\hline Previous myocardial infarction & $20,607(19.5 \%)$ & $18,275(18.5 \%)$ & $2332(33.3 \%)$ & $<0.001$ \\
\hline Ischemic heart disease & $31,523(29.8 \%)$ & $27,659(28.0 \%)$ & 3864 (55.1\%) & $<0.001$ \\
\hline Peripheral vascular disease & $9756(9.2 \%)$ & $8095(8.2 \%)$ & $1661(23.7 \%)$ & $<0.001$ \\
\hline Previous stroke & $11,419(10.8 \%)$ & $10,060(10.2 \%)$ & $1359(19.4 \%)$ & $<0.001$ \\
\hline Atrial fibrillation & $13,087(12.4 \%)$ & $10,980(11.1 \%)$ & $2107(30.0 \%)$ & $<0.001$ \\
\hline \multicolumn{5}{|l|}{ Medications use } \\
\hline Aspirin & $34,982(33.0 \%)$ & $31,334(31.7 \%)$ & $3648(52.0 \%)$ & $<0.001$ \\
\hline Statin & $44,690(42.2 \%)$ & $40,014(40.5 \%)$ & $4676(66.7 \%)$ & $<0.001$ \\
\hline Beta blockers & $31,845(30.1 \%)$ & $28,248(28.6 \%)$ & $3597(51.3 \%)$ & $<0.001$ \\
\hline ACE-i/ARBS & $39,413(37.2 \%)$ & $35,105(35.5 \%)$ & $4308(61.4 \%)$ & $<0.001$ \\
\hline Diuretics & $21,975(20.8 \%)$ & $18,930(19.1 \%)$ & $3045(43.4 \%)$ & $<0.001$ \\
\hline \multicolumn{5}{|l|}{ Echocardiography } \\
\hline $\operatorname{LVEDD}(\mathrm{cm})$ & $4.60 \pm 057$ & $4.60 \pm 0.56$ & $4.60 \pm 0.67$ & 0.975 \\
\hline $\operatorname{LVESD}(\mathrm{cm})$ & $2.84 \pm 0.64$ & $2.84 \pm 0.63$ & $2.98 \pm 0.76$ & $<0.001$ \\
\hline IVS (cm) & $1.01 \pm 0.23$ & $0.99 \pm 0.22$ & $1.24 \pm 0.20$ & $<0.001$ \\
\hline $\mathrm{PW}(\mathrm{cm})$ & $0.94 \pm 0.19$ & $0.92 \pm 0.18$ & $1.14 \pm 0.19$ & $<0.001$ \\
\hline LV MASS (gr) & $156.9 \pm 60.15$ & $152.76 \pm 58.00$ & $209.22 \pm 62.00$ & $<0.001$ \\
\hline \multicolumn{5}{|l|}{ As severity } \\
\hline Severe AS & & & $4125(58.8 \%)$ & \\
\hline Moderate AS & & & $2888(41.2 \%)$ & \\
\hline \multicolumn{5}{|l|}{ Aortic valve area $\left(\mathrm{cm}^{2}\right)$} \\
\hline Mean \pm SD & & & $0.94 \pm 0.28$ & \\
\hline Median (IQR) & & & $0.90(0.70-1.13)$ & \\
\hline \multicolumn{5}{|l|}{ Max aortic velocity (m/s) } \\
\hline Mean \pm SD & & & $3.53 \pm 0.83$ & \\
\hline Median (IQR) & & & $3.46(2.93-4.09)$ & \\
\hline \multicolumn{5}{|l|}{ Mean aortic gradient $(\mathrm{mmHg})$} \\
\hline Mean \pm SD & & & $32.0 \pm 17.6$ & \\
\hline Median (IQR) & & & $29.0(20.0-42.0)$ & \\
\hline
\end{tabular}

$A C E-i / A R B$ s angiotensin converting enzyme inhibitors/angiotensin II receptor blockers, $A S$ aortic stenosis, IVS interventricular septum, $L V$ left ventricular, LVEDD left ventricular end diastolic diameter, LVESD left ventricular end systolic diameter, PW posterior wall, SES socioeconomic class

${ }^{a}$ SES was missing in 1957 (1.8\%) patients 
Table 2 Crude and adjusted incidence rate of non-hematological cancers according to AS status and age

\begin{tabular}{|c|c|c|c|c|c|}
\hline AS status & $\begin{array}{l}\text { Events } \\
\text { (n) }\end{array}$ & $\begin{array}{l}\text { Follow-up } \\
\text { (person-years) }\end{array}$ & $\begin{array}{l}\text { Incidence rate } \\
\text { (Per } 1000 p-y)\end{array}$ & Crude HR $(95 \% \mathrm{Cl})$ & Adjusted HR ${ }^{\mathrm{a}}(95 \% \mathrm{Cl})$ \\
\hline \multicolumn{6}{|c|}{ Entire study population $(n=105,897)$} \\
\hline Mod or Severe AS $(n=7013)$ & 677 & 29,800 & 22.7 & $1.58(1.46-1.71)$ & $0.93(0.86-1.01)$ \\
\hline No AS $(n=98,884)$ & 10,028 & 733,067 & 13.7 & Reference & Reference \\
\hline \multicolumn{6}{|c|}{ Age lowest quartile: $20-49.7$ years $(n=26,476)$} \\
\hline Mod or Severe AS $(n=183)$ & 12 & 1330 & 9.0 & $2.26(1.28-3.99)$ & $1.91(1.08-3.39)$ \\
\hline No AS $(n=26,293)$ & 887 & 223,466 & 4.0 & Reference & Reference \\
\hline \multicolumn{6}{|c|}{ Age second quartile: $49.7-63$ years $(n=26,477)$} \\
\hline Mod or Severe AS $(n=558)$ & 58 & 3820 & 15.2 & $1.24(0.95-1.60)$ & $1.15(0.88-1.49)$ \\
\hline No AS $(n=25,919)$ & 2689 & 218,792 & 12.3 & Reference & Reference \\
\hline \multicolumn{6}{|c|}{ Age third quartile: $63-74.7$ years $(n=26,475)$} \\
\hline Mod or Severe AS $(n=1610)$ & 176 & 8345 & 21.1 & $1.02(0.87-1.18)$ & $0.96(0.82-1.12)$ \\
\hline No AS $(n=24,865)$ & 3575 & 175,370 & 20.4 & Reference & Reference \\
\hline \multicolumn{6}{|c|}{ Age highest quartile: $>74.7$ years $(n=26,469)$} \\
\hline Mod or Severe AS $(n=4662)$ & 431 & 16,306 & 26.4 & $1.00(0.91-1.11)$ & $0.99(0.89-1.10)$ \\
\hline No AS $(n=21,807)$ & 2877 & 115,438 & 24.9 & Reference & Reference \\
\hline
\end{tabular}

${ }^{a}$ Adjusted for age (continuous variable), sex, ethnicity, socioeconomic status, smoking, alcohol abuse, obesity, diabetes mellitus, previous history of cancer and aspirin and statin use

$\mathrm{Cl}$ confidence interval, $H R$ hazard ratio, $p$-y patient-year

between BAV and cancer in patients without any degree of AS $(n=98,884)$. Overall, BAV was found in 629 (0.6\%) subjects with no-AS compared to 534 (7.6\%) with AS. The presence of BAV without AS was not associated with an increased risk of cancer (HR 1.05, 95\% CI, 0.79-1.40).

We performed a sensitivity analysis to account for possible biases. First, we performed further adjustment for heart failure, ischemic heart disease, peripheral vascular disease, previous stroke, atrial fibrillation and year of study entry. This analysis showed similar results with significantly higher risk of cancer in the lowest age quartile (age 20-49.7 years), adjusted HR 1.86, 95\% CI 1.04-3.30 (Table 3). Second, we restricted the analysis to patients without a history of cancer, since these patients may have an increased risk for other malignancies (Table 3). Patients with AS at the lowest age quartile had a significantly increased risk for cancer even after exclusion of prior cancer. Third, we did landmark analysis to assess a possible surveillance bias shortly after AS diagnosis, by excluding patients with a cancer diagnosis during the first year after the index echo (Table 3). In this analysis the magnitude of the effect of AS remained unchanged, but did not reach statistical significance.

Distribution of the different types of cancer in patients with and without AS is presented in Table 4.

\section{Discussion}

In the present study, young patients in the lowest age quartile (20-49.7 years), who had moderate or severe AS, had a significantly higher adjusted risk of cancer compared to patients without AS. In addition, we have shown that age interacts with AS and modifies the risk for non-hematological malignancies. The risk of cancer associated with AS was inversely proportional to age. Our findings reconfirm our previous report in which the data was derived from a different study population [9]. In our earlier study, a post hoc analysis showed a significantly increased adjusted risk of non-hematological malignancies in younger patients with AS (40-60 years old), but not in older patients.

A cause-and-effect relationship between early cardiac remodeling secondary to left ventricular pressure overload and cancer was established using the TAC mice model [9]. Compared to controls, TAC-operated mice developed larger tumors after subcutaneous and fatpad injection of lung and breast cancer cells. In addition, compared to sham operated animals, TAC operated mice showed enhanced tumor metastasis after intravenous injection of tumor cells. Periostin was identified as a putative mediator of this effect [9]. Periostin is a nonstructural extracellular matrix protein secreted from the remodeled heart and associated with cancer progression and metastasis $[17,18]$. Periostin mRNA levels in the 
(a)

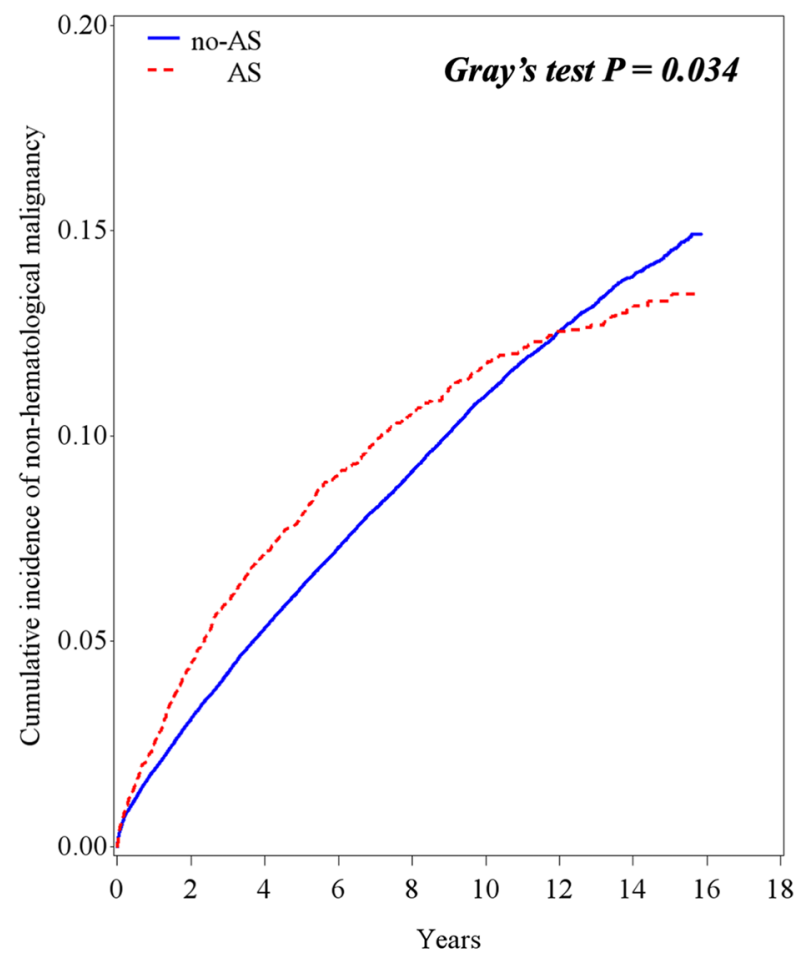

(b)

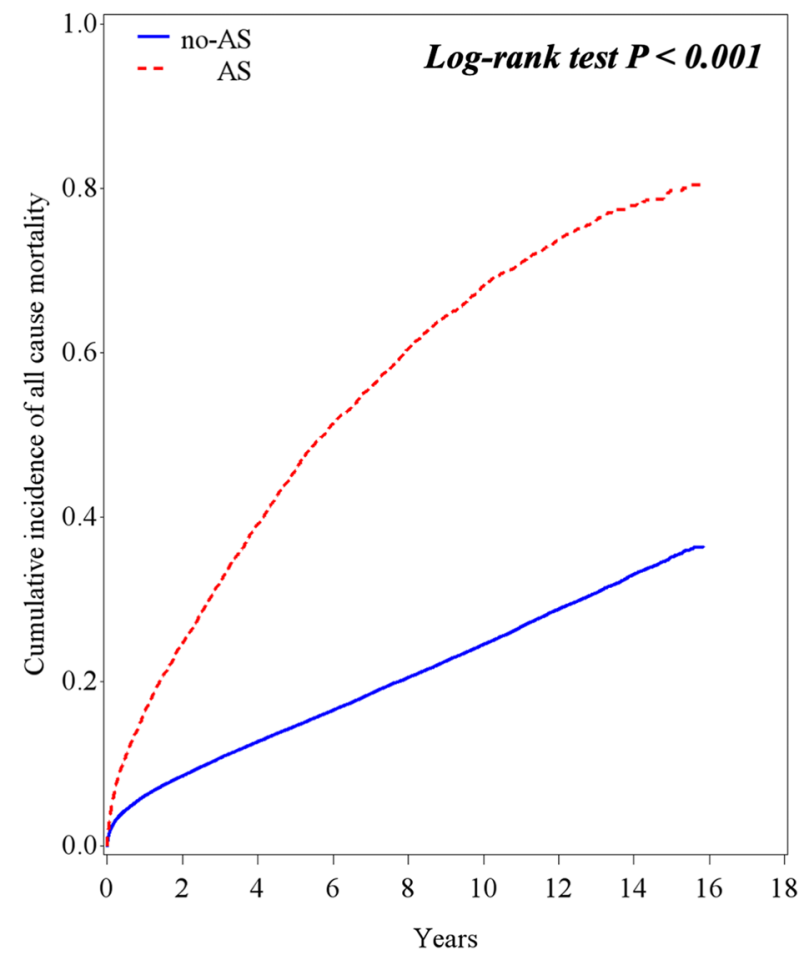

Fig. 2 Incidence of cancer and mortality in patients with and without AS. Cumulative incidence function (CIF) for the distribution of time to non-hematological malignancies (a) and Kaplan-Meier curves for mortality (b). Death was considered a competing event for cancer (a). Solid line represents patients without AS and dotted line patients with AS

heart increased after TAC, resulting high serum periostin levels. In vitro, serum from TAC-operated mice induced cancer cells proliferation, but depletion of periostin from the serum abrogated this effect. The effect of periostin on cancer progression needs to be confirmed in vivo.

It is not clear why the risk of cancer attributable to AS is most notable early in life and decreases with age. The incidence of both AS and cancer increases with age $[19,20]$. In a population study from Tromsø, Norway, the prevalence of AS (defined as aortic mean gradient $\geq 15 \mathrm{mmHg}$ ) was $0.2 \%$ in the $50-59$ year cohort, and increased exponentially with age to $9.8 \%$ in the 80-89 year cohort [21]. This phenomenon is due to the ongoing process of lipid deposition, inflammation and calcification of the aortic valve [22]. The incidence of invasive cancer peaks at age 70 but declines with advanced age (US population 2009 data) [19]. Cellular senescence can suppress cancer early in life, but later in life it may promote hyperplastic pathology and cancer by the secretion of chemokines and growth factors from senescent cells, possibly through the promotion of chronic inflammation [23]. The effect of tumor suppression by cellular senescence could be more dominant at advanced age, causing the decline in the incidence of cancer late in life. It is also possible, that while cell senescence and other disease processes promoting cancer are dominant in older age, periostin secretion as a result of cardiac adaptive remodeling secondary to AS is more dominant in younger age.

We performed a sensitivity analysis, by excluding patients with a history of cancer that could be at a higher risk for recurrent cancer. Young patients with AS had a significantly higher adjusted risk for cancer even after excluding patients with prior cancer. To exclude a surveillance bias, we excluded from the analysis patients in whom cancer was diagnosed during the first year after the diagnosis of AS. Albeit not reaching statistical significance, the magnitude of the effect of AS on cancer risk remained unchanged in younger age, arguing against a surveillance bias.

While most patients with AS above age 70 have a tricuspid aortic valve, almost all patients with AS younger than 50 have congenital AS, usually BAV [24]. It is 


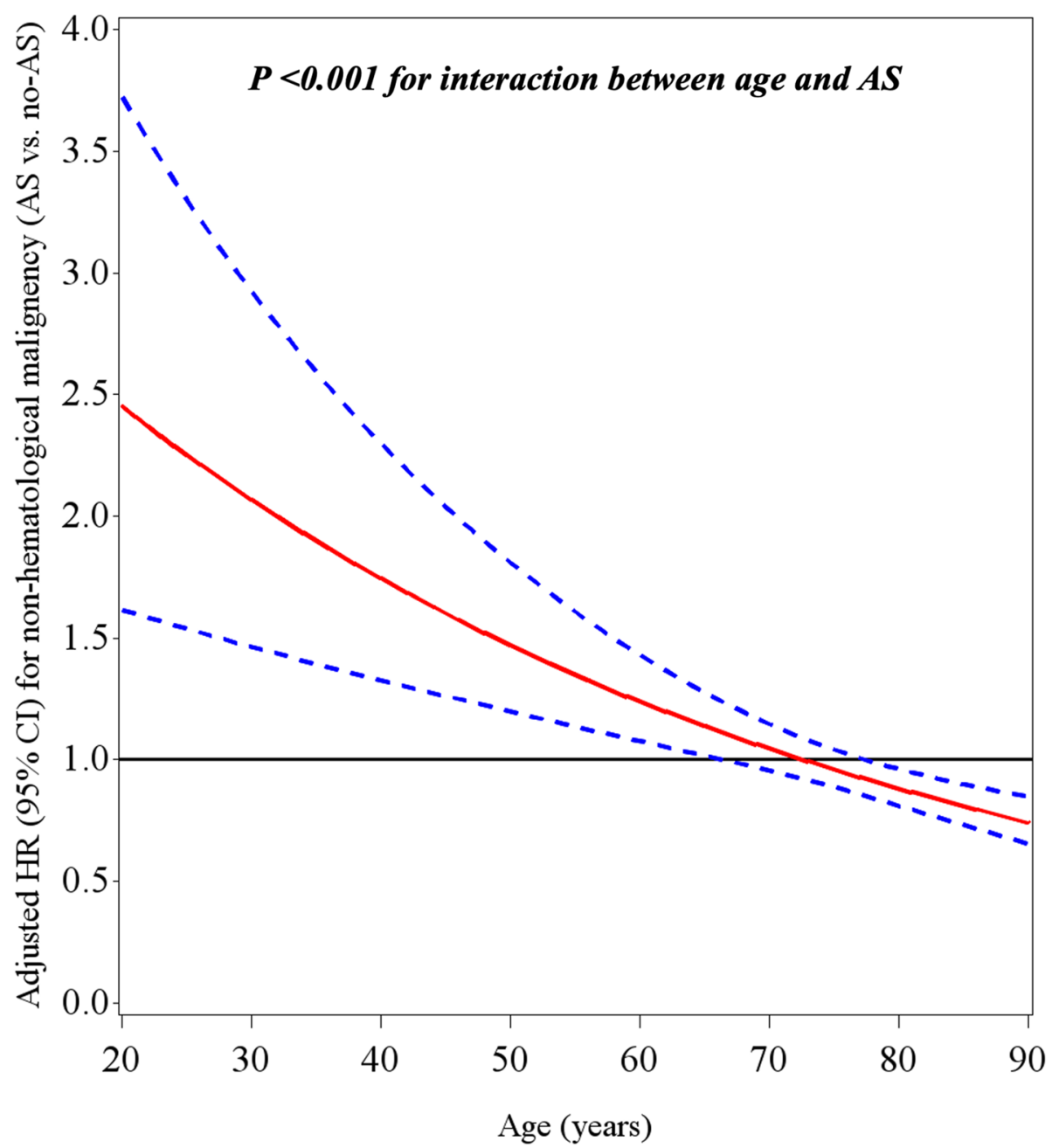

Fig. 3 The interaction between age and AS and the risk of cancer. Adjusted HR* for non-hematological malignancies in AS as a function of age. Dotted lines represent the $95 \%$ confidence interval (Cl). ${ }^{*}$ Adjusted for age (continuous variable), sex, ethnicity, socioeconomic status, smoking, alcohol abuse, obesity, diabetes mellitus, previous history of cancer and aspirin and statin use. $\mathrm{HR}=$ hazard ratio

possible that the increased risk of cancer is associated with BAV, but to the best of our knowledge there are no reports of such association. Furthermore, in our study, no association was found between BAV and cancer in patients without AS. Furthermore, the TAC mice model argues in favor of pressure overload secondary to AS and adaptive cardiac remodeling as the cause of cancer.

Our findings may suggest a closer cancer surveillance approach in young patients with AS, however the impact of such an approach on patient outcome is unknown. Early surgery in young patients with severe AS may decrease the risk for cancer, but should be weighed against the inherent risks of a mechanical prosthetic valve in this age group, mainly bleeding and thromboembolic complications $[25,26]$.

\section{Limitations}

In this study we rely on data retrieved from a computerized database originally designed for purposes of administrative and clinical management and not specifically designed for the present study. Yet, the database is periodically updated with data from the Israeli National Cancer Registry (INCR), which is highly accurate [13]. Based on the existence of strict criteria and standardized echocardiographic methodology for AS assessment, we estimate that the data on AS is highly accurate. Hence, it is likely that misclassification of the two main study variables (AS and cancer) is small. 


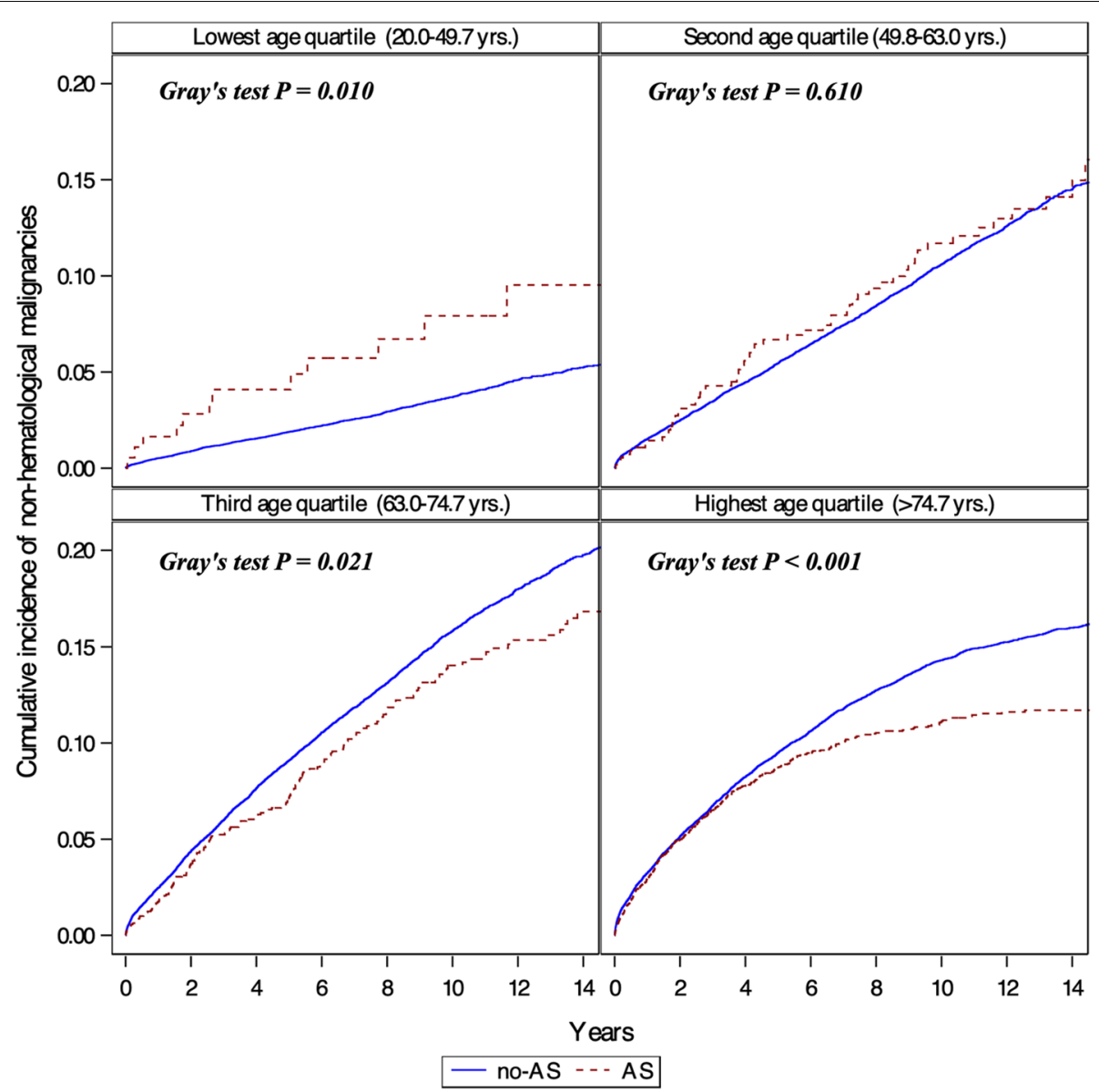

Fig. 4 Incidence of cancer by age. Cumulative incidence function (CIF) for the distribution of time to non-hematological malignancies stratified by age quartiles. Death was considered a competing event for cancer. Increased incidence of cancer is evident only in the lowest age quartile. Solid line represents patients with no AS and dotted lines represent patients with AS

Table 3 Sensitivity analysis displaying multivariable ${ }^{a}$ hazard ratios (HRs), stratified by age quartiles

\begin{tabular}{|c|c|c|c|}
\hline Age quartiles & $\begin{array}{l}\text { Adjustment } \\
\text { for additional } \\
\text { variables }^{b} \\
(n=105,897) \\
\text { HR }(95 \% \mathrm{Cl})\end{array}$ & $\begin{array}{l}\text { Patients without } \\
\text { prior malignancy } \\
(n=87,172) \\
\text { HR }(95 \% \mathrm{Cl})\end{array}$ & $\begin{array}{l}\text { One year } \\
\text { landmark } \\
\text { analysis }^{d} \\
(n=95,930) \\
\text { HR }(95 \% \mathrm{Cl})\end{array}$ \\
\hline $\begin{array}{l}\text { Lowest quartile } \\
(20-49.7 \text { years) }\end{array}$ & $1.86(1.04-3.30)$ & $2.24(1.06-4.71)$ & $1.71(0.88-3.31)$ \\
\hline $\begin{array}{l}\text { Second quartile } \\
\text { (49.7-63 years) }\end{array}$ & $1.14(0.88-1.48)$ & $0.91(0.63-1.32)$ & $1.22(0.93-1.62)$ \\
\hline $\begin{array}{l}\text { Third quartile } \\
\text { (63-74.7 years) }\end{array}$ & $0.95(0.82-1.11)$ & $0.96(0.79-1.16)$ & $1.03(0.88-1.22)$ \\
\hline $\begin{array}{l}\text { Highest quartile } \\
\text { (>74.7years) }\end{array}$ & $1.02(0.92-1.13)$ & $0.96(0.85-1.09)$ & $1.02(0.90-1.16)$ \\
\hline
\end{tabular}

${ }^{a}$ Adjusted for age (continuous variable), sex, ethnicity, socioeconomic status, smoking, alcohol abuse, obesity, diabetes mellitus, previous history of cancer (except for patients without prior malignancy analysis) and aspirin and statin use

${ }^{b}$ In addition to the above variables, additional adjustment was performed for heart failure, ischemic heart disease, peripheral vascular disease, previous stroke, atrial fibrillation, and year of study entry

cWithout prior malignancy of any type (hematological and non-hematological)

${ }^{d}$ The landmark analysis excluded patients with cancer diagnosed in the first year and was restricted to patients with follow up greater than one year
In addition, our study was limited to patients referred for echocardiography in a single tertiary medical center, and therefore includes a selected population, limiting the generalizability of our findings. In order to determine the risk for cancer in young patients with AS in a prospective population study, a very large sample size will be needed. The sample size of our study, together with the adjustment for a large number of confounders, and the concordance with our previous publication which was based on a different large database from another medical center, suggests that our findings are valid. Yet, due to the observational nature of our study residual confounders remain a major concern. We could not control for other potential confounders such as chronic inflammation, radiation, immunosuppression and diet which are known risk factors for cancer and might differ between subjects with and without AS.

In the current study we have focused on the association between AS and non-hematological 
Table 4 Distribution of invasive non-hematological malignancies

\begin{tabular}{lll}
\hline $\begin{array}{l}\text { Invasive non-hematological } \\
\text { malignancies type }\end{array}$ & No AS & AS \\
\hline $\begin{array}{l}\text { Lowest age quartile: 20-49.7years } \\
\text { Breast }\end{array}$ & $230(25.9 \%)$ & $3(25.0 \%)$ \\
Prostate & $17(1.9 \%)$ & $0(0 \%)$ \\
$\quad$ Gastrointestinal tract & $83(9.4 \%)$ & $2(16.7 \%)$ \\
Lung & $62(7.0 \%)$ & $0(0 \%)$ \\
Pancreas, liver and biliary tract & $41(4.6 \%)$ & $0(0 \%)$ \\
Kidney and bladder & $59(6.7 \%)$ & $1(8.3 \%)$ \\
Ovary, uterus and cervix & $89(10.0 \%)$ & $1(8.3 \%)$ \\
Melanoma & $47(5.3 \%)$ & $1(8.3 \%)$ \\
Thyroid & $65(7.3 \%)$ & $2(16.7 \%)$ \\
Other and unspecified site & $194(21.9 \%)$ & $2(16.7 \%)$ \\
All other patients, age > 49.7years & & \\
Breast & $1144(12.5 \%)$ & $56(8.4 \%)$ \\
Prostate & $951(10.4 \%)$ & $54(8.1 \%)$ \\
Gastrointestinal tract & $1505(16.5 \%)$ & $137(20.6 \%)$ \\
Lung & $1105(12.1 \%)$ & $71(10.7 \%)$ \\
Pancreas, liver and biliary tract & $702(7.7 \%)$ & $62(9.3 \%)$ \\
Kidney and bladder & $1153(12.6 \%)$ & $99(14.9 \%)$ \\
Ovary, uterus and cervix & $442(4.8 \%)$ & $24(3.6 \%)$ \\
Melanoma & $507(5.5 \%)$ & $44(6.6 \%)$ \\
Thyroid & $179(2.0 \%)$ & $4(0.6 \%)$ \\
Other and unspecified site & $1453(15.9 \%)$ & $114(17.1 \%)$ \\
\hline
\end{tabular}

malignancies. Our findings may not apply to hematological malignancies.

\section{Conclusions}

Our study suggests that younger patients with moderate or severe AS may have an increased risk of non-hematological malignancies. The risk of cancer associated with AS appears to be inversely proportional to age. These data are consistent with our previous findings from a different database, and together with the murine TAC model results may suggest that AS increases the risk of cancer.

\section{Acknowledgements}

Not applicable.

\section{Authors' contributions}

W.S and A.S. designed the study and drafted the manuscript. T.B. and W.S. analyzed the data. W.S. performed the statistical analysis. Y.S., S.S., A.S., M.V., S.A., A.F. and A.A. made critical revision of the manuscript. All authors read and approved the final manuscript.

\section{Authors' information}

Not applicable.

\section{Funding}

Israel Science Foundation ISF \# 731/17 to A.A.

\section{Availability of data and materials}

The datasets used and/or analyzed during the current study are available from the corresponding author on reasonable request.

\section{Declarations}

Ethics approval and consent to participate

The study was approved by the Institutional Review Board (IRB) and conducted in accordance with the Declaration of Helsinki.

\section{Consent for publication}

Not applicable.

\section{Competing interests}

The authors declare that they have no competing interests.

\section{Author details}

${ }^{1}$ Department of Community Medicine and Epidemiology, Lady Davis Carmel Medical Center, Haifa, Israel. ${ }^{2}$ The Ruth and Bruce Rappaport Faculty of Medicine, Technion, Israel Institute of Technology, Haifa, Israel. ${ }^{3}$ Department of Cardiology, Rabin Medical Center, Petah Tikva, Israel. ${ }^{4}$ Department of Cardiology, Lady Davis Carmel Medical Center, 7 Michal Street, 3436212 Haifa, Israel. ${ }^{5}$ Cell Biology and Cancer Science, Technion, Israel Institute of Technology, Haifa, Israel.

Received: 19 July 2021 Accepted: 13 October 2021

Published online: 25 October 2021

\section{References}

1. Aboumsallem JP, Moslehi J, de Boer RA. Reverse cardio-oncology: Cancer development in patients with cardiovascular disease. J Am Heart Assoc. 2020;9:1-12.

2. Meijers WC, De Boer RA. Common risk factors for heart failure and cancer. Cardiovasc Res. 2019;115:844-53.

3. Moslehi J, Zhang Q, Moore KJ. Crosstalk between the heart and cancer: beyond drug toxicity. Circulation. 2020;142:684-7.

4. Hasin T, Gerber Y, McNallan SM, Weston SA, Kushwaha SS, Nelson TJ, et al. Patients with heart failure have an increased risk of incident cancer. J Am Coll Cardiol. 2013;62:881-6.

5. Hasin T, Gerber Y, Weston SA, Jiang R, Killian JM, Manemann SM, et al. Heart failure after myocardial infarction is associated with increased risk of Cancer. J Am Coll Cardiol. 2016;68:265-71.

6. Banke A, Schou M, Videbæk L, Møller JE, Torp-Pedersen C, Gustafsson F, et al. Incidence of cancer in patients with chronic heart failure: a longterm follow-up study. Eur J Heart Fail. 2016;18:260-6.

7. Meijers WC, Maglione M, Bakker SJL, Oberhuber R, Kieneker LM, De Jong $\mathrm{S}$, et al. Heart failure stimulates tumor growth by circulating factors. Circulation. 2018;138:678-91.

8. Koelwyn GJ, Newman AAC, Afonso MS, van Solingen C, Corr EM, Brown $E J$, et al. Myocardial infarction accelerates breast cancer via innate immune reprogramming. Nat Med Springer US. 2020;26:1452-8.

9. Avraham S, Abu-Sharki S, Shofti R, Haas T, Korin B, Kalfon R, et al. Early cardiac remodeling promotes tumor growth and metastasis. Circulation. 2020;142:670-83.

10. Kezerle L, Tsadok MA, Akriv A, Senderey AB, Bachrach A, Leventer-Roberts $\mathrm{M}$, et al. Pre-diabetes increases stroke risk in patients with Nonvalvular atrial fibrillation. J Am Coll Cardiol. 2021;77:875-84.

11. Artzi NS, Shilo S, Hadar E, Rossman H, Barbash-Hazan S, Ben-Haroush A, et al. Prediction of gestational diabetes based on nationwide electronic health records. Nat Med. 2020;26:71-6.

12. Dagan N, Barda N, Kepten E, Miron O, Perchik S, Katz MA, et al. BNT162b2 mRNA Covid-19 vaccine in a Nationwide Mass vaccination setting. N Engl J Med. 2021;384:1412-23.

13. Moore E, Silverman BG, Fishier Y, Ben-Adiva E, Davidov O, Dichtiar R, et al. An assessment of the completeness and timeliness of the Israel national cancer registry. Isr Med Assoc J. 2021;23:23-7.

14. Mitchell C, Rahko PS, Blauwet LA, Canaday B, Finstuen JA, Foster $M C$, et al. Guidelines for performing a comprehensive transthoracic 
echocardiographic examination in adults: recommendations from the American Society of Echocardiography. J Am Soc Echocardiogr Elsevier. 2019;32:1-64.

15. Baumgartner H, Hung J, Bermejo J, Chambers JB, Edvardsen T, Goldstein $\mathrm{S}$, et al. Recommendations on the echocardiographic assessment of aortic valve stenosis: a focused update from the European Association of Cardiovascular Imaging and the American Society of Echocardiography. J Am Soc Echocardiogr Elsevier Inc. 2017;30:372-92.

16. Gooley TA, Leisenring W, Crowley J, Storer BE. Estimation of failure probabilities in the presence of competing risks: new representations of old estimators. Stat Med. 1999;18:695-706.

17. Litvin J, Zhu S, Norris R, Markwald R. Periostin family of proteins: therapeutic targets for heart disease. Anat Rec Part A Discov Mol Cell Evol Biol. 2005;287:1205-12.

18. Ruan K, Bao S, Ouyang G. The multifaceted role of periostin in tumorigenesis. Cell Mol Life Sci. 2009;66:2219-30.

19. White MC, Holman DM, Boehm JE, Peipins LA, Grossman M, Jane HS. Age and cancer risk: a potentially modifiable relationship. Am J Prev Med. 2014;46:S7-15.

20. Nkomo VT, Gardin JM, Skelton TN, Gottdiener JS, Scott CG, EnriquezSarano M. Burden of valvular heart diseases: a population-based study. Lancet. 2006;368:1005-11.

21. Eveborn GW, Schirmer H, Heggelund G, Lunde P, Rasmussen K. The evolving epidemiology of valvular aortic stenosis. The Tromsø Study. Heart. 2013;99:396-400.

22. Otto CM, Kuusisto J, Reichenbach DD, Gown AM, O'Brien KD. Characterization of the early lesion of 'degenerative' valvular aortic stenosis: histological and immunohistochemical studies. Circulation. 1994;90:844-53.

23. Campisi J. Aging, cellular senescence, and cancer. Annu Rev Physiol. 2013;75:685-705

24. Roberts WC, Ko JM. Frequency by decades of unicuspid, bicuspid, and tricuspid aortic valves in adults having isolated aortic valve replacement for aortic stenosis, with or without associated aortic regurgitation. Circulation. 2005;111:920-5.

25. Hammermeister K, Sethi GK, Henderson WG, Grover FL, Oprian C, Rahimtoola SH. Outcomes 15 years after valve replacement with a mechanical versus a bioprosthetic valve: final report of the veterans affairs randomized trial. J Am Coll Cardiol Elsevier Masson SAS. 2000;36:1152-8.

26. Stassano P, Di Tommaso L, Monaco M, lorio F, Pepino P, Spampinato N, et al. Aortic Valve Replacement. A Prospective Randomized Evaluation of Mechanical Versus Biological Valves in Patients Ages 55 to 70 Years. J Am Coll Cardiol Elsevier Inc. 2009:54:1862-8.

\section{Publisher's Note}

Springer Nature remains neutral with regard to jurisdictional claims in published maps and institutional affiliations.
Ready to submit your research? Choose BMC and benefit from:

- fast, convenient online submission

- thorough peer review by experienced researchers in your field

- rapid publication on acceptance

- support for research data, including large and complex data types

- gold Open Access which fosters wider collaboration and increased citations

- maximum visibility for your research: over $100 \mathrm{M}$ website views per year

At BMC, research is always in progress.

Learn more biomedcentral.com/submissions 